Note

\title{
A Novel Chromogenic Screening Medium for Isolation of Enterohemorrhagic Escherichia coli
}

\author{
HAJIME TERAMURA, JUN-ICHIRO SEKIGUCHI, \\ AND KOHAKU INOUE \\ Microbiology Research Division, Kohjin Bio Co., Ltd., \\ 5-1-3 Chiyoda, Sakado, Saitama 350-0214, Japan
}

Received 24 October, 2012/Accepted 13 January, 2013

\begin{abstract}
EHEC-chrom, a novel chromogenic screening agar medium for enterohemorrhagic Escherichia coli (EHEC), was developed. A total of 52 EHEC strains, which were studied for the inclusivity study, grew and formed blue-green colored colonies on EHEC-chrom. When 43 gram-negative bacteria other than EHEC were inoculated for the exclusivity study, 10 strains grew and formed colorless colonies. A total of 28 gram-positive bacteria failed to grow and 1 yeast strain grew as colorless colonies.

EHEC-chrom was compared with CHROMagar ${ }^{T M}$ STEC, XM-EHEC agar and CT-MacConkey base agar with a specific sugar (CT-SorbitoIMAC, CT-rhamnoseMAC and CT-sorboseMAC) as commercially available selective agar using 100 food samples artificially contaminated with low levels ( $<10$ logCFU/25g) of EHEC. Numbers of samples from which EHEC was recovered by using EHEC-chrom, CHROMagar ${ }^{\text {TM }}$ STEC, XM-EHEC agar and CT-MacConkey base agar with specific sugar were $62,58,59$ and 60 , respectively. Our results suggested EHEC-chrom was a useful alternative for EHEC screening in food.
\end{abstract}

Key words : Enterohemorrhagic Escherichia coli/EHEC/Chromogenic medium.

Enterohemorrhagic Escherichia coli (EHEC) is known as an important food borne pathogen that can cause diarrhea, bloody diarrhea and haemolytic uraemic syndrome through the consumption of contaminated food of various kinds (Griffin and Tauxe, 1991). Hence, the control of EHEC is a very important issue for the prevention of food poisoning. In Japan, the largest $E$. coli $O 157$ outbreak occurred by the ingestion of radish sprouts as the presumed source of contamination in Osaka in 1996 (Michino et al., 1999). Since then, although EHEC contamination has been a matter of great concern in food safety, a large E. coli 0111 outbreak also occurred through the consumption of raw beef (yukhoe) in Toyama Prefecture in 2011. Serotype 0157 has been the most commonly implicated strain in food poisoning cases, however, other serotypes of EHEC than 0157 such as $\mathrm{O} 26$ and $\mathrm{O} 111$ have been also reported as the causa-

*Corresponding author. Tel : +81-49-284-3781, Fax : +8149-284-4784, E-mail: teramura (a)kohjin-bio.co.jp tive agents (Bettelheim, 2007; Bradley et al., 2012; Buchholz et al., 2011; Ethelberg et al., 2009; Tzschoppe et al., 2012).

The conventional sorbitol MacConkey agar with cefixime and potassium tellurite (CT-SMAC) can detect only EHEC serotype 0157 (Zadik et al., 1993). Similarly, CT-SMAC with rhamnose or sorbose as substitute for sorbitol can detect only either serotype 026 or 0111 (Caro et al., 2011). Hence, several types of selective media are needed for screening EHEC. Meanwhile, several chromogenic selective screening media for EHEC such as CHROMagar ${ }^{\mathrm{TM}}$ STEC (CST; CHROMagar Microbiology, Paris, France) and XM-EHEC agar (XME; Nissui Pharmaceutical Co., Ltd., Tokyo, Japan), have been developed (Bettelheim, 2005; Manafi and Kremsmaier, 2001; Tzschoppe et al., 2012). However, most of the current commercially available chromogenic selective media detect only a specific serotype of EHEC. As a result, EHEC-chrom (ECA; Kohjin Bio Co., Ltd., Saitama, Japan) has been 
developed as novel chromogenic selective agar for the isolation of EHEC regardless of serotype.

ECA was developed primarily to be selective for EHEC. ECA contains the following ingredients per liter: casein peptone (Scharlau, Scharlab SL, Barcelona, Spain), 10.0 g; lab-lemco powder (Oxoid Limited, Hampshire, UK), $2.5 \mathrm{~g}$; yeast extract, $1.0 \mathrm{~g}$; cellobiose (Nippon Paper Chemicals, Co., Ltd., Tokyo, Japan), 10.0 g; lactose, 2.5 g; sodium cholate, $0.4 \mathrm{~g}$; sodium deoxycholate, $0.6 \mathrm{~g}$; potassium tellurite (Wako Pure Chemical Industries, Ltd., Osaka, Japan), 2.5 mg; cefixime (Astellas Pharma Inc., Tokyo, Japan), 0.05mg, 5-Bromo-4-Chloro-3-Indolyl$\beta$-D-Galactopyranoside (X-GAL; Biosynth AG, Staad, Switzerland), $0.1 \mathrm{~g}$; and agar (SSK sales Co., Ltd., Shizuoka, Japan), $15.0 \mathrm{~g}$ (pH 7.4). After $24 \pm 2$ $\mathrm{h}$ incubation at $36 \pm 1{ }^{\circ} \mathrm{C}$, EHEC grew and formed blue-green colored colonies (Fig. 1). The aim of this study was to evaluate the ECA for its performance in the detection and isolation of EHEC.

Fifty-two strains of EHEC including 33 strains of E. coli O157: H7 were inoculated for the inclusivity study. For the exclusivity study, 43 gram-negative strains other than EHEC, 28 gram-positive strains and 2 yeasts were inoculated. After each tested bacterial strain and yeast strain were cultured in Tryptic Soy Broth (Difco, Becton Dickinson, Detroit, MI, USA) at $36 \pm 1{ }^{\circ} \mathrm{C}$ for $24 \pm 2 \mathrm{~h}$ and Sabouraud Dextrose Broth (Difco) at $25 \pm 1{ }^{\circ} \mathrm{C}$ for $72 \pm 3 \mathrm{~h}$, respectively, each loopful of culture was streaked onto ECA, CST and $\mathrm{XME}$, respectively. The growth and colony color of each tested strain were read after $24 \pm 2 \mathrm{~h}$ incubation at $36 \pm 1^{\circ} \mathrm{C}$.

Results from both the inclusivity and the exclusivity

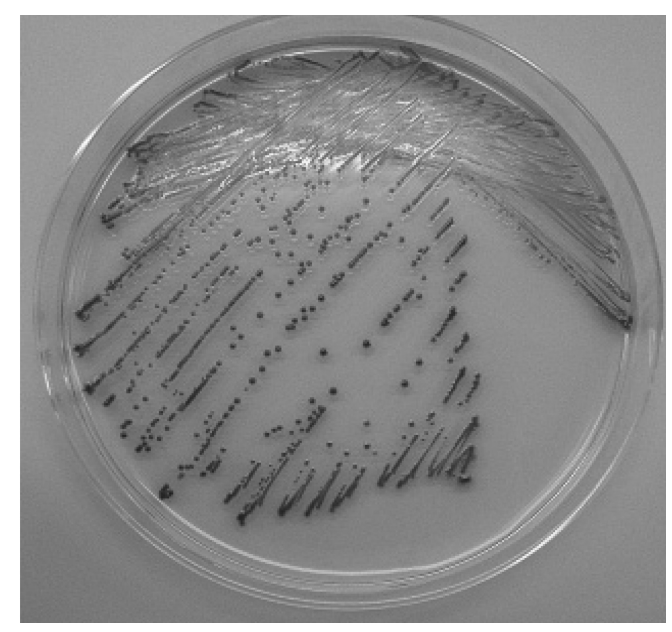

FIG. 1. Typical colonies of E. coli O157: H7 formed on EHEC-chrom. E. coli 0157: H7 ATCC 35150 grew and formed blue-green colored colonies on EHEC-chrom after $24 \pm 2 \mathrm{~h}$ of incubation at $36 \pm 1^{\circ} \mathrm{C}$. serotype of EHEC, a total of 52 EHEC strains grew and formed typical blue-green and mauve colored colonies on ECA and CST, respectively. On the other hand, 32 of $33 E$. coli 0157 strains and all tested $E$. coli $\mathrm{O} 111$ and $\mathrm{O} 118$ strains grew and formed magenta colored colonies, and all tested E. coli O26, O103, O113, O119, O145, O146 and O161 grew and formed blue colored colonies on XME. However, 1 strain of $E$. coli $O 157$ failed to grow on XME. Of the 43 gram-negative strains other than EHEC, E. hermannii, Proteus mirabilis, Pseudomonas spp., Salmonella enterica and Serratia fonticola grew as colorless colonies on ECA. On the other hand, S. fonticola grew as blue colored colonies on CST, and E. hermannii and $S$. fonticola were detected as false positive magenta colored colonies on XME. A total of 28 grampositive bacteria failed to grow and 1 yeast strain grew as colorless colonies on all tested media.

Regarding the makeup of the media, CST contains a fluorescence substrate for discrimination of $E$. coli O157. Hence, CST contains 3 kinds of chromogenic substrates at least for the detection and elimination of other bacteria. Moreover, XME contains sorbitol and 2 kinds of chromogenic substrates for $\beta$-galactosidase and $\beta$-glucuronidase for the differentiation of E. coli O157, O26 and O111. Thus, ECA was suggested as a more cost-effective screening medium since ECA contains only $X-G A L$ as a chromogenic substrate. Moreover, ECA had a good selectivity through the combination of $\mathrm{X}-\mathrm{GAL}$ and cellobiose in addition to 2 kinds of bile salts, cefixime and potassium tellurite. However, the addition of a small amount of lactose was necessary for the bluegreen color development of EHEC. Addition of IPTG (isopropylthiogalactoside) for induction of $\beta$-galactosidase of EHEC did not lead to the bluegreen color development of EHEC. It seemed EHEC did not use X-GAL directly in the presence of cellobiose. Of the coliform bacteria, only $E$. coli including EHEC could not use cellobiose. Since ECA contained cellobiose at 4 times the amount of lactose, it seemed that microbes other than E. coli used cellobiose preferentially. Moreover, it seemed X-GAL was degraded in parallel when $E$. coli used lactose. As a result, it seemed ECA had selective color development for EHEC.

Our results show that media which could presumptively discriminate 3 major serotypes such as $\mathrm{XME}$, could not assuredly differentiate other EHEC since EHEC other than those 3 major serotype grew as colonies of various colors. Hence, the determination of serotype by colony color makes no sense since all EHEC must be controlled for food safety.

ECA was compared with CST, XME and CT- 
TABLE 1. Growth and colony color of microbes cultivated on various media.

\begin{tabular}{|c|c|c|c|c|c|c|c|c|c|}
\hline & \multicolumn{3}{|c|}{ No. of tested strains } & \multicolumn{6}{|c|}{ No. of strains grown ${ }^{b}$} \\
\hline & Standard $^{a}$ & Isolate & Total & & & & & & \\
\hline $\begin{array}{l}\text { EHEC }^{\mathrm{c}} \\
\text { E. coli O157: } \mathrm{H} 7 \\
\text { E. coli O26: } \mathrm{H}- \\
\text { E. coli O26: } \mathrm{H} 11 \\
\text { E. coli O103: } \mathrm{H} 25 \\
\text { E. coli O111: } \mathrm{H}- \\
\text { E. coli O113: } \mathrm{H} 4 \\
\text { E. coli O118: } \mathrm{H} 2 \\
\text { E. coli O119: } \mathrm{H} 7 \\
\text { E. coli O119: } \mathrm{H} 25 \\
\text { E. coli O145: } \mathrm{H}- \\
\text { E. coli O146: } \mathrm{H} 19 \\
\text { E. coli O161: } \mathrm{H}- \\
\text { Subtotal }\end{array}$ & $\begin{array}{l}2 \mathrm{~A} \\
0 \\
0 \\
0 \\
0 \\
0 \\
0 \\
0 \\
0 \\
0 \\
0 \\
0 \\
2\end{array}$ & $\begin{array}{c}31 \\
1 \\
6 \\
1 \\
3 \\
1 \\
1 \\
1 \\
1 \\
2 \\
1 \\
1 \\
50\end{array}$ & $\begin{array}{c}33 \\
1 \\
6 \\
1 \\
3 \\
1 \\
1 \\
1 \\
1 \\
2 \\
1 \\
1 \\
52\end{array}$ & $\begin{array}{l}33 \\
1 \\
6 \\
1 \\
3 \\
1 \\
1 \\
1 \\
1 \\
2 \\
1 \\
1 \\
52\end{array}$ & $\begin{array}{l}(B G) \\
(B G) \\
(B G) \\
(B G) \\
(B G) \\
(B G) \\
(B G) \\
(B G) \\
(B G) \\
(B G) \\
(B G) \\
(B G)\end{array}$ & $\begin{array}{c}33 \\
1 \\
6 \\
1 \\
3 \\
1 \\
1 \\
1 \\
1 \\
2 \\
1 \\
1 \\
52\end{array}$ & $\begin{array}{l}\text { (m) } \\
\text { (m) } \\
(\mathrm{m}) \\
(\mathrm{m}) \\
(\mathrm{m}) \\
(\mathrm{m}) \\
(\mathrm{m}) \\
(\mathrm{m}) \\
(\mathrm{m}) \\
(\mathrm{m}) \\
(\mathrm{m}) \\
(\mathrm{m})\end{array}$ & $\begin{array}{c}32 \\
1 \\
6 \\
1 \\
3 \\
1 \\
1 \\
1 \\
1 \\
2 \\
1 \\
1 \\
51\end{array}$ & $\begin{array}{l}\text { (M) } \\
\text { (B) } \\
\text { (B) } \\
\text { (B) } \\
\text { (M) } \\
\text { (B) } \\
\text { (BM) } \\
\text { (B) } \\
\text { (B) } \\
\text { (B) } \\
\text { (B) } \\
\text { (B) }\end{array}$ \\
\hline
\end{tabular}

Gram negative bacteria except for EHEC Aeromonas hydrophila

Achromobacter denitrificans

A. xylosoxidans

Alcaligenes faecalis

Citrobacter freundii

C. koseri

Enterobacter aerogenes

E. amnigenus

E. cloacae

E. intermedius

E. sakazakii

Escherichia blattae

E. coli (non-pathogenic)

E. fergusonii

E. hermannil

Hafnia alvei

Klebsiella oxytoca

K. ozaenae

K. pneumoniae

Kluyvera ascorbata

K. cryocrescens

Morganella morganii

Proteus mirabilis

$P$ vulgaris

Pseudomonas aeruginosa

$P$. putida

Rahnella aquatilis

Salmonella enterica

Serratia fonticola

S. liquefaciens

S. marcescens

Shigella flexineri

S. sonnei

Subtotal

\begin{tabular}{|c|c|c|}
\hline J & 0 & 1 \\
\hline $1 \mathrm{~J}$ & 0 & 1 \\
\hline $1 \mathrm{~J}$ & 0 & 1 \\
\hline $1 \mathrm{~J}$ & 0 & 1 \\
\hline $1 \mathrm{~A}$ & 0 & 1 \\
\hline $1 \mathrm{~A}$ & 0 & 1 \\
\hline $1 \mathrm{~A}$ & 0 & 1 \\
\hline $1 \mathrm{~A}$ & 0 & 1 \\
\hline $1 \mathrm{~A}$ & 0 & 1 \\
\hline $2 \mathrm{~A}$ & 0 & 2 \\
\hline $1 \mathrm{~A}$ & 0 & 1 \\
\hline $1 \mathrm{~J}$ & 0 & 1 \\
\hline $4 \mathrm{~A}$ & 0 & 4 \\
\hline $2 \mathrm{~J}$ & 0 & 2 \\
\hline $1 \mathrm{~A}$ & 0 & 1 \\
\hline $1 \mathrm{~A}$ & 0 & 1 \\
\hline $1 \mathrm{~A}$ & 0 & 1 \\
\hline $1 \mathrm{~A}$ & 0 & 1 \\
\hline $1 \mathrm{~A}$ & 0 & 1 \\
\hline $1 \mathrm{~A}$ & 0 & 1 \\
\hline $1 \mathrm{~A}$ & 0 & 1 \\
\hline $1 \mathrm{~A}$ & 0 & 1 \\
\hline $1 \mathrm{~A}$ & 0 & 1 \\
\hline $1 \mathrm{~A}$ & 0 & 1 \\
\hline $4 \mathrm{~A}$ & 0 & 4 \\
\hline $1 \mathrm{~A}$ & 0 & 1 \\
\hline 1J & 0 & 1 \\
\hline $2 \mathrm{~A}$ & 0 & 2 \\
\hline 1A & 0 & 1 \\
\hline $1 \mathrm{~A}$ & 0 & 1 \\
\hline $2 \mathrm{~A}$ & 0 & 2 \\
\hline 0 & 1 & 1 \\
\hline $1 \mathrm{~A}$ & 0 & 1 \\
\hline 4 & 1 & 43 \\
\hline
\end{tabular}

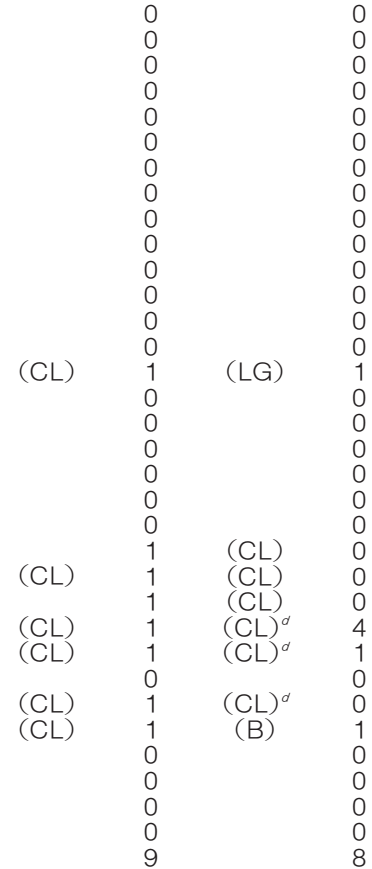

(M)

$(\mathrm{CL})$

(M)

Gram positive bacteria

Bacillus cereus

$B$. licheniformis

B. subtilis

Corynebacterium minutissimum

C. renale

C. xerosis

Enterococcus avium

E. durans

E. faecalis

E faecium

Micrococcus luteus

Staphylococcus aureus

S. auricularis

S. capitis

S. epidermidis

S. haemolyticus

S. hominis

S. lentus

S. saprophyticus

S. sciuri

S. simulans

S. warneri

S. xylosus

Subtota

$\begin{array}{llll}1 A & 0 & 1 & 0 \\ 1 A & 0 & 1 & 0 \\ 1 A & 0 & 1 & 0 \\ 1 A & 0 & 1 & 0 \\ 1 A & 0 & 1 & 0 \\ 1 A & 0 & 1 & 0 \\ 1 A & 0 & 1 & 0 \\ 1 A & 0 & 1 & 0 \\ 1 A & 0 & 1 & 0 \\ 1 A & 0 & 1 & 0 \\ 1 A & 0 & 1 & 0 \\ 4 A & 1 & 5 & 0 \\ 1 A & 0 & 1 & 0 \\ 1 A & 0 & 1 & 0 \\ 2 A & 0 & 2 & 0 \\ 1 A & 0 & 1 & 0 \\ 1 A & 0 & 1 & 0 \\ 1 A & 0 & 1 & 0 \\ 1 A & 0 & 1 & 0 \\ 1 A & 0 & 1 & 0 \\ 1 A & 0 & 1 & 0 \\ 1 A & 0 & 1 & 0 \\ 1 A & 0 & 1 & 0 \\ \text { 1A } & 1 & 28 & 0\end{array}$

0
0
0
0
0
0
0
0
0
0
0
0
0
0
0
0
0
0
0
0
0
0
0
0

Yeasts

Candida albicans

Subtotal

$$
\text { Total }
$$

$\begin{array}{ccc}0 & 2 & 1 \\ 0 & 2 & 1 \\ 52 & 125 & 63\end{array}$

(CL)

1
1
62

(CL)

(CL)

aStandard strains were from A; ATCC (American Type Culture Collection) and J; JCM (Japan Collection of Microorganisms).

${ }^{b}$ Parentheses indicate colony color: BG, blue-green; B, blue; M, magenta; LM, light-magenta; CL, colorless; m, mauve; LG, light-gray.

All tested EHEC were confirmed to produce verotoxins by immunochromatography for VT1 or 2

( $\mathrm{NH}$ immunochromato VT1/2; Nippon Meat Packers, Inc.)

${ }^{\circ}$ Grown only at the site of high density streaking. 
MacConkey base agar with a specific sugar using artificially contaminated food samples. One-hundred samples (12 beef samples, 37 pork samples, 15 chicken samples, 6 ham samples, 18 leafy vegetable samples and 12 sprout samples) were purchased from retail stores in Saitama. For confirmation that these samples were negative for EHEC, after being homogenized with mEC broth (Nissui Pharmaceutical Co., Ltd.) for $2 \mathrm{~min}$ by a homogenizer (Pro-media SH001, ELMEX LIMITED, Tokyo, Japan), samples were incubated for $24 \pm 2 \mathrm{~h}$ at $42 \pm 1^{\circ} \mathrm{C}$. Each loopful of cultured $\mathrm{mEC}$ broth was then transferred onto ECA, CST and $\mathrm{XME}$, respectively. For the comparison experiments, three strains of EHEC (E. coli O157 ATCC 43890 (American Type Culture Collection, Manassas, VA, USA), E. coli O26 and E. coli O111 derived from clinical specimens) were used randomly for inoculation. After each $25 \mathrm{~g}$ sample was inoculated at $<10$ CFU $/ 25 \mathrm{~g}$ level, samples were stored at $4^{\circ} \mathrm{C}$ for 3 days. Each artificially contaminated sample was added to a 9-fold volume of mEC broth and was homogenized for $2 \mathrm{~min}$. After $24 \pm 2 \mathrm{~h}$ enrichment at 42 $\pm 1{ }^{\circ} \mathrm{C}$, each sample was streaked onto ECA, CST, $\mathrm{XME}$ and CT-SMAC (Nissui Pharmaceutical Co., Ltd.) for 0157 or CT-rhamnoseMAC (Nissui Pharmaceutical Co., Ltd.) for O26 or CT-sorboseMAC (Nissui Pharmaceutical Co., Ltd.) for O111, respectively. After $24 \pm 2 \mathrm{~h}$ incubation at $36 \pm 1^{\circ} \mathrm{C}$, five typical colonies for EHEC on each tested media were picked up and identified using the API 20E system (SYSMEX bioMerieux Co., Ltd., Tokyo, Japan). Confirmation for 0157, O26 and O111 was conducted using each O antiserum (Denka Seiken Co., Ltd., Tokyo, Japan), respectively.

Table 2 shows the number of samples in which EHEC was recovered by using each medium (recovered sample number). The recovered sample numbers of ECA, CST, XME and CT-MacConkey base agar with a specific sugar were $62,58,59$ and 60 , respectively. The detection rate of ECA was highest among the tested media in this study. Although ECA recovered EHEC, CST (4 samples), XME (2 samples) and CT-MacConkey base agar with specific sugar (2 samples) failed to do so. In CST and XME, there was a possibility that these false negatives were resulted from overlapping growth of grown bacteria other than EHEC. Moreover, false negative results on CT-MacConkey base agar with a specific sugar were likely to be caused by the reddish coloration of a colorless EHEC colony by other bacteria. Manafi and Kremsmaier (2001) showed many Enterobacteriaceae strains, grown on CT-SMAC as red colored colony, that used sorbitol. Hence, in case that many Enterobacteriaceae strains grew near the colony of EHEC, usage of sugar by Enterobacteriaceae caused the possibility of reddish coloration of an EHEC colony since the CTMacConkey agar base contained $\mathrm{pH}$ indicator dye.

On the contrary, there were false positive results on ECA (1 sample) and XME (3 samples). As for their identification, the false positive strain on ECA was Klebsiella pneumoniae and the false positive strains on XME were Enterobacter cloacae, Escherichia hermannii and Klebsiella oxytoca. However $K$. pneumoniae on ECA, E. cloacae and K. oxytoca on $\mathrm{XME}$ could be discriminated by colony appearance

TABLE 2. Summary of detection of EHEC in artificially contaminated food samples by using various selective media

\begin{tabular}{|c|c|c|c|c|c|c|c|c|c|}
\hline \multirow[b]{2}{*}{ Spiked strain } & \multirow[b]{2}{*}{$n$} & & \multicolumn{7}{|c|}{ Number of samples (\%) } \\
\hline & & & ECA & CST & XME & CT-SMAC & CT-SBMAC & CT-RMAC & $\begin{array}{l}\text { CT-MAC base } \\
\text { with sugar }\end{array}$ \\
\hline \multirow[t]{2}{*}{ E. coli 0157} & 34 & positive & $13(38.2)$ & $13(38.2)$ & $13(38.2)$ & $11(32.4)$ & NT & NT & \\
\hline & & negative & $21(61.8)$ & $21(61.8)$ & $21(61.8)$ & $23(67.6)$ & NT & NT & \\
\hline \multirow[t]{2}{*}{ E. coli 0111} & 33 & positive & $21(63.6)$ & $19(57.6)$ & $19(57.6)$ & NT & $21(63.6)$ & NT & \\
\hline & & negative & $12(36.4)$ & $14(42.4)$ & $14(42.4)$ & NT & $12(36.4)$ & NT & \\
\hline \multirow[t]{2}{*}{ E. coli $\mathrm{O} 26$} & 33 & positive & $28(84.8)$ & $26(78.8)$ & $27(81.8)$ & NT & NT & $28(84.8)$ & \\
\hline & & negative & $5(15.2)$ & $7(21.2)$ & $6(17.6)$ & NT & NT & $5(15.2)$ & \\
\hline \multirow[t]{2}{*}{ Total } & 100 & positive & $62(62.0)$ & $58(58.0)$ & $59(59.0)$ & - & - & - & $60(60.0)$ \\
\hline & & negativ & $38(38.0)$ & $42(42.0)$ & $41(41.0)$ & - & - & - & $40(40.0)$ \\
\hline
\end{tabular}

Number of samples from which EHEC was recovered by using each medium in 100 artificially contaminated food samples. ECA; EHEC-chrom, CST; CHROMagar STEC, XME; XM-EHEC, CT-SMAC; CT-Sorbitol MacConkey for O157, CT-SBMAC; CT-Sorbose MacConkey for O111, CT-RMAC; CT-Rhamnose MacConkey for O26. NT; Not tested.

${ }^{2}$ Total of CT-SMAC, CT-SBMAC and CT-RMAC. 
since these were observed as whitish mucoid colonies.

Although the CT-MacConkey base agar with specific sugar including CT-SMAC have been used for detecting EHEC for a long time, the colony color of EHEC on these media is susceptible to $\mathrm{pH}$ change caused by usage of sugar by other bacteria. Thus, in case that a sample contains many other bacteria, there is a possibility of false negative results. Moreover, these methods have high costs since several kinds of media which contain different sugars need to be used depending on the serotypes. On the other hand, chromogenic media such as ECA, CST and XME can more efficiently distinguish EHEC from other bacteria since EHEC forms a distinctive colored colony. Moreover, these media are beneficial since one kind of medium can detect several kinds of serotype of EHEC. In food and food processing facilities, all EHEC regardless of the serotype must be controlled. Thus, the ECA, chromogenic agar media able to distinguish a wider range of EHEC serotypes, is more efficient for food safety.

In conclusion, ECA is excellent for the selectivity and differentiation of EHEC. Therefore, ECA is a suitable alternative for the screening EHEC in food safety control.

\section{ACKNOWLEDGMENT}

We are grateful to Yumiko Itabashi and Harumi Yajima (Kohjin Bio Co., Ltd.) for their technical assistance and to Dr. Stephan Speidel (HyServe GmbH \& Co. KG) for his critical reading of the text.

\section{REFERENCES}

Bettelheim, K. A. (2005) Reliability of 0157: H7 ID agar (0157 H7 ID-F) for the detection and isolation of verotoxigenic strains of Escherichia coli belonging to serogroup 0157. J. Appl. Microbiol., 99, 408-410.

Bettelheim, K. A. (2007) The non-0157 shiga-toxigenic (verocytotoxigenic) Escherichia coli; under-rated pathogens. Crit. Rev. Microbiol., 33, 67-87.

Bradley, K. K., Williams, J. M., Burnsed L. J., Lytle, M. B., McDermott, M. D., Mody, R. K., Bhattarai, A., Mallonee, S., Piercefield, E. W., McDonald-Hamm, C. K., and Smithee, L. K. (2012) Epidemiology of a large restaurantassociated outbreak of shiga toxin-producing Escherichia coli O111: NM. Epidemiol. Infect., 140, 1644-1654.

Buchholz, U., Bernard, H., Werber, D., Böhmer, M. M., Remschmidt, C., Wilking, H., Deleré, Y., ander Heiden, M., Adlhoch, C., Dreesman, J., Ehlers, J., Ethelberg, S., Faber, M., Frank, C., Fricke, G., Greiner, M., Höhle, M., Ivarsson, S., Jark, U., Kirchner, M., Koch, J., Krause, G., Luber, P., Rosner, B., Stark, K., and Kühne, M. (2011) German outbreak of Eschrichia coli O104: H4 associated with sprouts. N. Engl. J. Med., 365, 1763-1770.

Caro, I., Mateo, J., Rúa, J., and Del Rosario García-Armesto, M. (2011) Occurrence of Escherichia coli O157, O111 and 026 in raw ewe's milk and performance of two enrichment broths and two plating media used for its assessment. Int. J. Food Microbiol., 146, 84-87.

Ethelberg, S., Smith, B., Torpdahl, M., Lisby, M., Boel, J., Jensen, T., Nielsen, E. M., and Moolbak, K. (2009) Outbreak of non-O157 shiga toxin-producing Escherichia coli infection from consumption of beef sausage. Clin. Infect. Dis., 48, 78-81.

Griffin, P. M., and Tauxe, R. V. (1991) The epidemiology of infections caused by E.coli O157: H7, other enterohemorrhagic E. coli and the associated hemolytic uremic syndrome. Epidemiol. Rev., 13, 60-98.

Manafi, M., and Kremsmaier, B. (2001) Comparative evaluation of different chromogenic/fluorogenic media for detecting Escherichia coli 0157: H7 in food. Int. J. Food Microbiol., 71, 257-262.

Michino, H., Araki, K., Minami, S., Takaya, S., Sakai, N., Miyazaki, M., Ono, A. and Yanagawa, H. (1999) Massive outbreak of Escherichia coli O157: H7 infection in schoolchildren in Sakai City, Japan, associated with consumption of white radish sprouts. Am. J. Epidemiol., 150, 787796.

Tzschoppe, M., Martin, A., and Beutin, L. (2012) A rapid procedure for the detection and isolation of enterohaemorrhagic Escherichia coli (EHEC) serogroup 026, 0103, 0111, 0118, 0121, 0145 and 0157 strains and the aggregative EHEC 0104: H4 strain from readyto-eat vegetables. Int. J. Food Microbiol., 152, 19-30.

Zadik, P. M., Chapman, P. A., and Siddons, C. A. (1993) Use of tellurite for the selection of verocytotoxigenic Escherichia coli 0157. J. Med. Microbiol., 39, 155-158. 